\title{
The Role of Stress on Charge Transfer through Self-Assembled Alkanethiol Monolayers on Au
}

\author{
K. -A. Son, H. I. Kim and J. E. Houston \\ Sandia National Laboratories, Albuquerque, NM 87185-1415
}

\begin{abstract}
We have studied charge-transfer through alkanethiol molecules self-assembled on $\mathrm{Au}(111)$ substrates using interfacial force microscopy. Simultaneous measurement of the tipsubstrate current and the normal interfacial force reveals the critical role of tip-film contact. Measurable currents are only seen for tip-applied stresses above about $20 \mathrm{Mpa}$, after which the current rises exponential with stress. We suggest that charge transfer results from stress-induced band-gap states near the Fermi level in these normally highly insulating molecular films.
\end{abstract}

Tunneling and other charge transfer processes in self-assembled monolayers (SAM) has have been studied extensively for molecular electronics applications, and molecules with highly delocalized electrons have demonstrated their potential use as electronic devices.[1-4] In contrast, alkanethiol molecules, which are wide-band insulators, have received considerably less attention. However, the scanning tunneling microscope (STM) has successfully produced molecular-level images of these insulating SAM films,[5-7] indicating that tunneling is possible. Calculations seem to support tunneling without tip-film contact,[8] although recent studies indicate that tunneling requires contact.[8,9] Thus, the degree of interaction is still controversial and the mechanisms responsible for the charge transfer have not been established.

To this end, we have investigated the details of the charge-transfer process for two alkanethiol SAM films, hexadecanethiol $\left(\mathrm{C}_{16}\right)$ and dodecanethiol $\left(\mathrm{C}_{12}\right)$, on $\mathrm{Au}(111)$ using the 


\section{DISCLAIMER}

This report was prepared as an account of work sponsored by an agency of the United States Government. Neither the United States Government nor any agency thereof, nor any of their employees, make any warranty, express or implied, or assumes any legal liability or responsibility for the accuracy, completeness, or usefulness of any information, apparatus, product, or process disclosed, or represents that its use would not infringe privately owned rights. Reference herein to any specific commercial product, process, or service by trade name, trademark, manufacturer, or otherwise does not necessarily constitute or imply its endorsement, recommendation, or favoring by the United States Government or any agency thereof. The views and opinions of authors expressed herein do not necessarily state or reflect those of the United States Government or any agency thereof. 


\section{DISCLAIMER}

Portions of this document may be illegible in electronic image products. Images are produced from the best available original document. 
interfacial force microscopy (IFM). This technique is similar to the atomic force microscope but is distinguished by its use of a mechanically stable, noncompliant and quantitative force sensor.[10,11] This sensor enables simultaneous measurements of conductance, normal force and lateral friction force over the entire range of tip/sample interaction and allows us to quantify the degree of interaction necessary to achieve significant charge transfer.

In our study, the alkanethiol SAMs were assembled by soaking a $\mathrm{Au}(111)$ substrate in a $0.5 \mathrm{mM}$ ethanol-based solution at room temperature. The substrate was produced from a $100 \mu \mathrm{m}$ Au wire by first cleaning it with a $3: 1 \mathrm{H}_{2} \mathrm{SO}_{4} / \mathrm{H}_{2} \mathrm{O}_{2}$ solution followed by flame annealing to produce a small sphere with several flat (111) facets.[12] The thiol assembly time was $~ 36$ hours for $\mathrm{C}_{12}$ and $\sim 15$ hours for the $\mathrm{C}_{16}$ molecules. After assembly, the sample was rinsed with ethanol and dried with dry $\mathrm{N}_{2}$. The Au probe tip was formed into an parabolic shape by electrochemical etching a $100 \mu \mathrm{m}$ Au wire in a $10: 1 \mathrm{H}_{2} \mathrm{SO}_{4} / \mathrm{H}_{2} \mathrm{O}_{2}$ solution followed by cleaning in acetone and isopropyl alcohol. Based on SEM images, the radius of the curvature for the $\mathrm{Au}$ tip used in this work was $\sim 3000 \AA$.

In IFM, the force-feedback sensor electrostatically balances the total torque about the sensor axis resulting from the force applied to the tip.[13] Lateral forces are distinguished from normal forces by applying a small $(\sim 12 \AA) 100 \mathrm{~Hz}$ lateral modulation between the tip and sample and separating the two signals in the frequency domain by lock-in detection.[14] Currents were measured with a self biasing current amplifier (Kfifthley Model 428) by holding the probe tip at ground potential and biasing the sample. Current vs. sample-bias voltage (IV) data were taken by establishing a set-point current, locking the feedback loop (thus holding a constant tip/sample separation) and sweeping the bias voltage while monitoring the junction current. 
One of the key questions that we were interested in answering was whether tunneling currents could be measured without disturbing the SAM film. We approached this question by measuring tip/substrate junction current and normal force as a function of tip displacement at the limit of our current detection capabilities $(\sim 10 \mathrm{fA})$. Figure 1 illustrates these results for both $\mathrm{C}_{12}$ and $\mathrm{C}_{16}$ films showing the current in $\mathrm{fA}$ and the normal force in $\mathrm{nN}$ plotted as a function of tip displacement. Attractive forces are indicated by negative force values. In order to increase the current sensitivity, these profiles were taken at large bias voltages ( 2.0 and $2.5 \mathrm{~V}$ for $\mathrm{C}_{12}$ and $\mathrm{C}_{16}$, respectively) and were averaged over three repetitive scans. The force curves are typical of a van der Waals interaction (along with a small electrostatic component) and film contact is signaled by the point of inflection where the force begins to turn towards repulsive values. This is the region where the attractive forces at the periphery of the contact are beginning to be balanced by the repulsive forces near the contact center. For both the $\mathrm{C}_{12}$ and $\mathrm{C}_{16}$ films, we observe no current in excess of the noise until the tip makes physical contact with the SAM surface. The current then increases exponentially with the relative displacement. This behavior was observed over the entire range of bias voltages sampled ( $1 \mathrm{mV}$ to $2.5 \mathrm{~V})$ and for considerably higher loads and currents. Therefore, it is clear that film contact is necessary for conductance through these two self-assembled films, in agreement with previous work taken with significantly less sensitivity.[8,9] The log slope of the current vs. displacement plot is approximately $-0.25 \AA^{-1}$ and $-0.31 \AA^{-1}$ for the $C_{12}$ and $C_{16}$ films, respectively. In the case of tunneling between the tip and a bare Au surface through vacuum gap, the log slope is approximately $-1 \AA^{-1} \cdot[15]$

As additional clues to the origin of charge transfer after contact, we have taken IV data as well as simultaneous measurements of normal force, lateral-friction force and current. A 
representative set of IV data for the $C_{16}$ film is shown in Fig. 2 for several initial set-point current values. The forces applied to the SAM at these set-point currents are in the repulsive region, as implied by the data of Fig.1. All the IV curves in Fig. 2 appear symmetric and show a reasonably linear slope at low bias voltages. The slope in this region defines the zero-bias conductance of the junction.[3] At higher bias values the current shows a nonlinear increase. An adequate fit to the curves is provided by a linear plus cubic function, as illustrated by the solid lines in Fig. 2.

The linear plus cubic fits from Fig. 2 indicate that the junction conductance, which is simply the derivative of the IV data and is a common way of representing tunneling behavior, takes the form of an offset parabola. The offset values represent the zero-bias conductance, which is found to increase exponentially with increasing set-point current, and the coefficients of the quadratic term scale reasonably well with the offsets. Finally, in Fig. 3 we show the threshold behavior of the current and lateral friction force as a function of relative tip displacement for the $\mathrm{C}_{16}$ film. It is clear from these data that the current and the friction force rise at the same relative tip/sample separation, strongly indicating a common source.

To set the stage for developing some understanding of these results, it is instructive to see what tunneling conductance would be expected based on simple models. For the $\mathrm{Au} / \mathrm{Au}$ system without the SAM film, the tunneling can be modeled from the relationship,[16]

$$
\mathrm{G}_{\mathrm{t}}=2 \times 10^{-5} \exp (-1.025 \sqrt{ } \varphi \mathrm{z}),
$$

where $G_{t}$ is in reciprocal ohms or siem qn $^{e}$ (S) and $z$ is the tip-substrate separation in $\AA$. $\varphi$ is the average barrier height, which was determined to be $\sim 4.7 \mathrm{eV}$.[16] The exponential coefficient of $\mathrm{z}$ (calculated to be $2.22 \AA^{-1}$ ) is very near the commonly quoted STM value of one decade per $\AA$ A.[15] 
Tunneling conductance in the presence of the film can be estimated using the "molecularwire" relationship discussed by Samanta, et al,[17] i.e.,

$$
\mathrm{G}_{\mathrm{f}}=78 \times 10^{-5} \exp \left(-1.025 \sqrt{\mathrm{E}_{\mathrm{g}}} / 2 \mathrm{z}\right),
$$

where the coefficient is the quantum unit of conductance $\left(2 \mathrm{e}^{2} / \mathrm{h}\right)$ and $\mathrm{E}_{\mathrm{g}}$ is the HOMO-LUMO gap ( $\sim 9 \mathrm{eV}$ for these molecules[18]). Equation (2) assumes that the Fermi energy is located at the center of the HOMO-LUMO gap, which is to be expected for the metal-molecule contact since these molecules are not able to take on significant charge.[17] Thus, the barrier height for tunneling $(\varphi)$ is just $E_{g} / 2$. At initial tip-film contact, the value of $z$ in Eq. (2) corresponds to the thickness of the film ( 18 and $\sim 22 \AA$ for the $\mathrm{C}_{12}$ and $\mathrm{C}_{16}$ films, respectively[19]). From Eq. (2), conductance values at this point are calculated to be $\sim 8 \times 10^{-21}$ and $\sim 1.3 \times 10^{-24} \mathrm{~S}$ for the $\mathrm{C}_{12}$ and $\mathrm{C}_{16}$ films, respectively. Since $\mathrm{E}_{\mathrm{g}} / 2$ for the films is close to the tunneling barrier for the $\mathrm{Au} / \mathrm{Au}$ system, similar conductance values are calculated at contact (differing only by the ratio of the coefficients, 39 ). Thus, the presence of the alkanethiol films would not be expected to enhance tunneling to the extent as to permit its experimental detection. This is in stark contrast to the calculations presented in conjunction with an earlier study,[8] which showed dramatic enhancements in tunneling current at contact due to the presence of the molecules. However, our conclusion is supported by experimental results from several previous studies, including the one just cited.[8,9] The question still remains, "What mechanism is responsible for the dramatic increase in conductance after contact?"

As a first step, it is instructive to estimate the level of stress imposed on the films and how the tunnel conductance varies as a result. We can approximately calculate the average stress by dividing the repulsive component of the after-contact force by the contact area. The area is determined from the simple contract-mechanics relationship $A=\pi R d$, where $R$ is the tip radius 
and $d$ is the film deformation after contact.[20] This result is shown in Fig. 4 for a $\mathrm{C}_{16}$ film taken with a bias of $1.2 \mathrm{mV}$. Here the conductance is plotted against the average stress in MPa. Since the contact area increases linearly with film deformation, we see that the conductance increases exponentially with stress, rising above our noise floor only after reaching stresses in excess of about $20 \mathrm{MPa}$.

Several authors have suggested the presence of molecular defects, e.g., gauche configurations, in SAM films under contact.[9,21,22] Figure 3 supports this suggestion, since the current and friction rise with essentially the same threshold. The presence of a frictional force means that energy is delivered to the molecules as a result of moving the contact area laterally, and creating defects would be an avenue for such processes.[23] In terms of tunneling, deviations from the all trans, ground-state configuration for the thiolate molecules would be expected to increase their total energy and decrease $E_{g}$, thus lowering the exponential coefficient in Eq. (2). This is in the direction implied by the exponential coefficients of Fig. 1. However, these values require $\mathrm{E}_{\mathrm{g}}$ to be less than $\sim 0.6 \mathrm{eV}$, which would place the molecular film in the semiconductor range. Other authors have suggested that stress may play a role in facilitating conductance in films under contact[7-9], presumably by HOMO-LUMO gap narrowing. However, gap narrowing does not appear adequate to explain our overall results. Since the gap would shrink with applied stress, and $\mathrm{E}_{\mathrm{g}}$ appears in the square-root term of Eq. (2), reducing its value with stress would be expected to give rise to a stress dependent $\mathrm{z}$ coefficient. However, we have taken data over many orders of magnitude in current and no significant deviation from the exponential relationship was observed.

The quadratic CV behavior implied by the data of Fig. 2 may offer valuable hints. In a simple square-barrier model, the application of a bias voltage reduces the effective barrier height 
by half the value of voltage applied.[15] Thus, conductance varies exponentially with bias, as observed in STM experiments[15] and calculations of CV behavior for tunneling through insulators.[24] Clearly, the CV behavior implied by the data of Fig. 2 does not fit these exponential predictions.

The small log slopes of Fig. 1, and the peculiar CV behavior implied by Fig. 2, suggest another explanation for our results. The applied stresses may narrow the band gap of the film to such an extent that the tails of the HOMO-LUMO states overlap. This would give rise to a more semi-metallic-like behavior, i.e., small densities of both filled and empty states at the Fermi level. The zero-bias conductance would then depend on the density of these Fermi-level states, which would be expected to increase as the bands broaden and further cross under added stress. In turn, the $\mathrm{CV}$ behavior would depend on the joint density-of-states of the band tails (i.e., the integral of the product of the filled and empty states over the extent of the overlap) and would be expected to have a much more gentle variation with bias, as observed. The quadratic variation in conductance with bias would further require that both sets of states increase linearly with energy near the Fermi level.

There are many observations of the kind of conductance behavior we have just described in high-pressure physics and chemistry. For example; (1) both insulator-to-metal and metal-toinsulator transitions have been documented in nanotubes under $100 \mathrm{MPa}$-level transverse stresses [25], (2) Si has long been known to have a semiconductor-to-metal transition at stresses near 12 Gpa.[26] and (3) Drickamer and coworkers have observed exponential resistance behavior in high-pressure studies on normally insulting polyacene molecular solids as a function of hydrostatic pressure.[27] All of these results were explained in a similar way to that suggested here. However, the final answer to the question as to the mechanisms responsible for the 
increased charge transfer for alkanethiol SAMs under stress will have to await detailed calculations addressing the stress-related changes in electronic structure for these hydrocarbon materials.

In conclusion, we have presented evidence that charge transfer through alkanethiol SAM films as short as $C_{12}$ is immeasurably small unless there is significant contact between the tip and film surface. The conductance after contact rises exponentially with tip displacement with decay lengths a factor of three to four smaller than those seen for tunneling in the absence of the SAM film. Stress levels in excess of $\sim 20 \mathrm{MPa}$ are found before measurable conductance is observed, after which the conductance rises exponentially with stress. The coincident rise of lateral friction and conductance after contact supports the creation of molecular defects. However, the CV data, as well as the small and constant exponential coefficients for the current vs. tip displacement, hint that a stress induced, semi-metallic conduction processes may be responsible for the observed results.

\section{Acknowledgement}

Sandia is a multiprogram laboratory operated by Sandia Corporation, a Lockheed Martin Company, for the U.S. Dept. of Energy under Contract DE-AC04-94AL85000. 


\section{Figure Captions}

Fig.1. Current and normal-force profiles (a) for a $C_{12}$ thiol under a $2.0 \mathrm{~V}$ bias and (b) for a $\mathrm{C}_{16}$ thiol with a bias of $2.5 \mathrm{~V}$. The solid lines indicate the approximate log slope of the current.

Fig. 2. Current vs. sample-bias voltage (IV) data taken for a $\mathrm{C}_{16}$ thiol at various current set points for a sample bias of $5 \mathrm{mV}$. The solid curves indicate the result of a least-square fit to a linear and cubic function.

Fig. 3. Simultaneous measurements of the tunneling current, with a sample bias of $100 \mathrm{mV}$, and the lateral friction force as a function of the relative tip displacement.

Fig. 4. The variation in the measured conductance for a $\mathrm{C}_{16}$ thiol film as a function of the calculated average-applied stress. The arrow indicates the approximate noise floor of our conductance measurements $\left(\sim 4 \times 10^{-11} \mathrm{~S}\right)$.

\section{References}

[1] M. A. Reed and J. M. Tour, Scientific American 282, 86 (2000).

[2] Molecular Electronics: Science and Technology, edited by A. Aviram and M. Ratner, Annals of the New York Academy of Sciences Vol. 852 (New York Academy of Sciences, New York, 1998).

[3] S. Datta, Electronic Transport in Mesoscopic Systems (Cambridge University Press, Cambridge, 1997), 
[4] C. Joachim and J. K. Gimzewski, Proc. IEEE 86, 184 (1998).

[5] G. E. Poirier and M. J. Tarlov, Langmuir 10, 2853 (1994).

[6] K. Kobayashi, T. Horiuchi, H. Yamada, and K. Matsushige, Thin Solid Films 331, 210 (1998).

[7] L. A. Bumm, J. J. Arnold, T. D. Dunbar, D. L. Allara, and P. S. Weiss, J. Phy. Chem. B 103, 8122 (1999).

[8] M. Salmeron, G. Neubauer, A. Folch, M. Tomitori, D. F. Ogletree, and P. Sautet, Langmuir 9, 3600 (1993).

[9] U. Durig, O. Zuger, B. Michel, L. Haussling, and H. Ringsdorf, Phys. Rev. B 48, 1711 (1993).

[10] J. E. Houston and T. A. Michalske, Nature 356, 266 (1992).

[11] S. A. Joyce and J. E. Houston, Review of Scientific Instruments 62, 710 (1991).

[12] U. Demir and C. Shannon, Langmuir 10, 2794 (1994).

[13] J. D. Kiely and J. E. Houston, Langmuir 15, 4513 (1999).

[14] H. I. Kim, V. Boiadjiev, J. E. Houston, X. -Y. Zhu, and J. D. Kiely, Tribol. Lett. , in press. (2000).

[15] J. A. Stroscio and R. M. Feenstra, in Scanning Tunneling Microscopy, edited by J. A. Stroscio and W. K. Kaiser, Methods of Experimental Physics, p. 95 (Academic Press, Inc., San Diego, CA, 1993).

[16] L. Olesen, M. Brandbyge, M. R. Sorensen, K. W. Jacobsen, E. Laegsgaard, I. Stensgaard, and F. Besenbacher, Phys. Rev. Lett. 76, 1485 (1996).

[17] M. P. Samanta, W. Tian, S. Datta, J. I. Henderson, and C. P. Kubiak, Phys. Rev. B 53, R7626 (1996). 
[18] A. Dhirani, P. H. Lin, P. Guyot-Sionnest, R. W. Zehner, and L. R. Sita, J. Chem. Phys. 106, $5249(1997)$.

[19] A. Ulman, An Introduction to Ultrathin Organic Films: from Langmuir-Blodgett to SelfAssembly (Academic Press, Inc., San Diego, 1991), p. 279.

[20] K. L. Johnson, Contact Mechanics (Cambridge University Press, Cambridge, 1985), p. 84.

[21] J. I. Siepmann and I. R. McDonald, Phys. Rev. Lett. 70, 453 (1993).

[22] K. J. Tupper, R. J. Colton, and D. W. Brenner, Langmuir 10, 2041 (1994).

[23] X. D. Xiao, J. Hu, D. H. Charych, and M. Salmeron, Langmuir 12, 235 (1996).

[24] M. Magoga and C. Joachim, Phys. Rev. B 56, 4722 (1997).

[25] P. E. Lammert, P. H. Zhang, and V. H. Crespi, Phys. Rev. Lett. 84, 2453 (2000).

[26] J. M. Mignot, G. Chouteau, and G. Martinez, Physica B \& C 135, 235 (1985).

[27] G. A. Samara and H. G. Drickamer, J. Chem. Phys. 37, 474 (1962). 


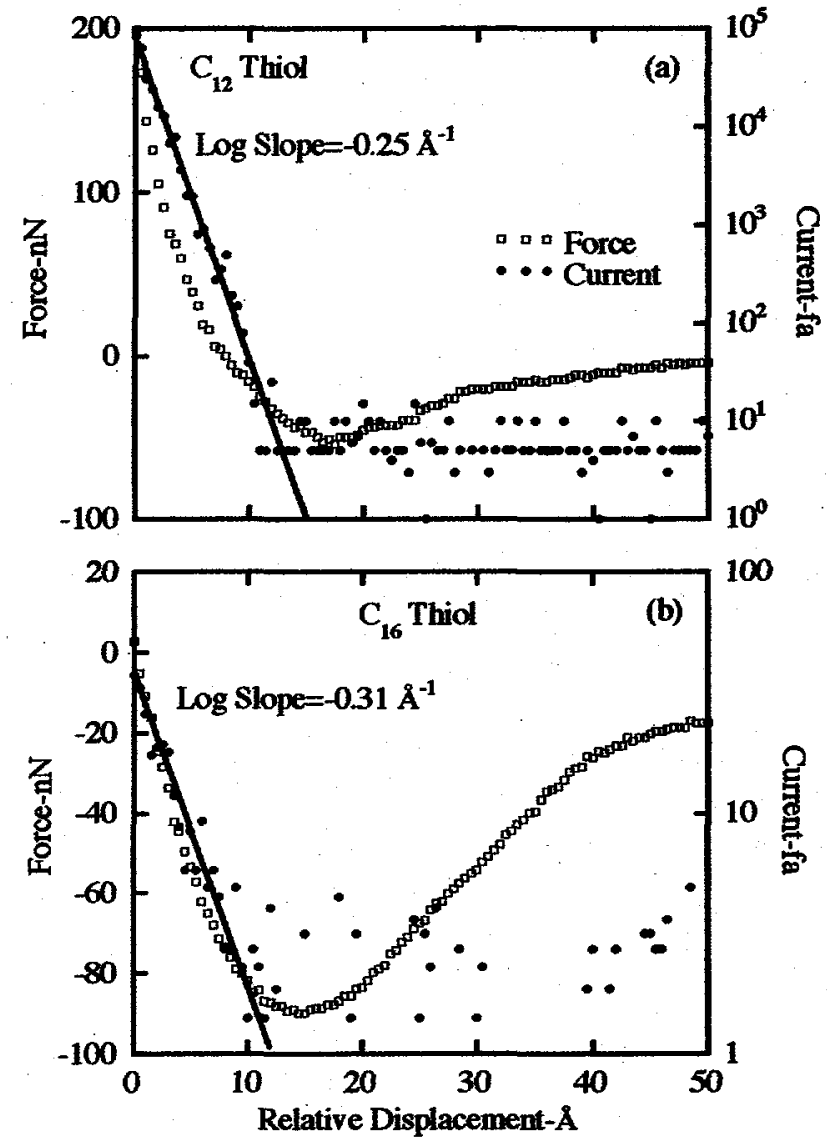

Fig. 1, Son, Kim \& Houston 


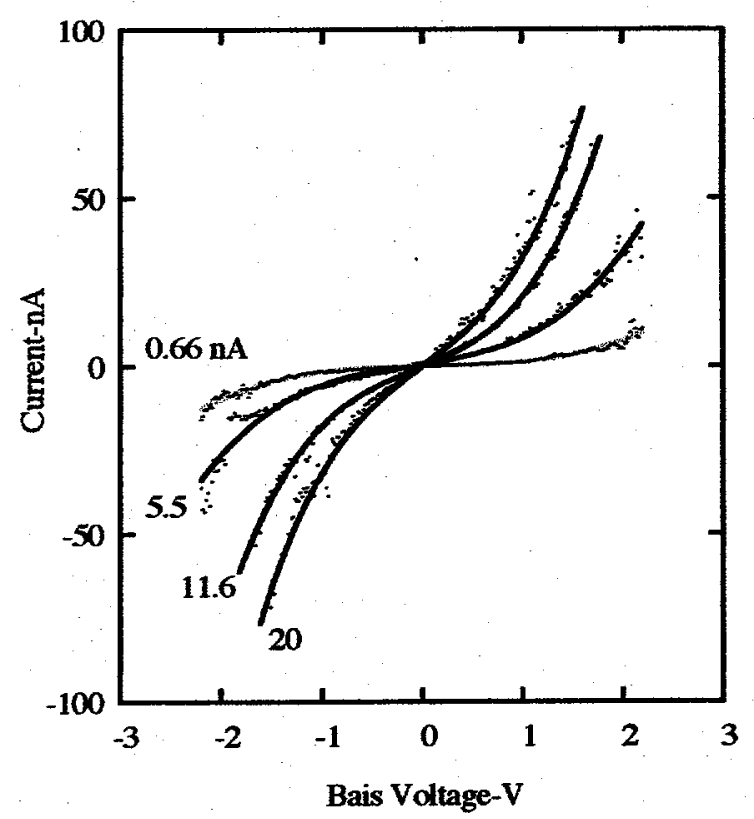

Fig. 2, Son, Kim \& Houston 


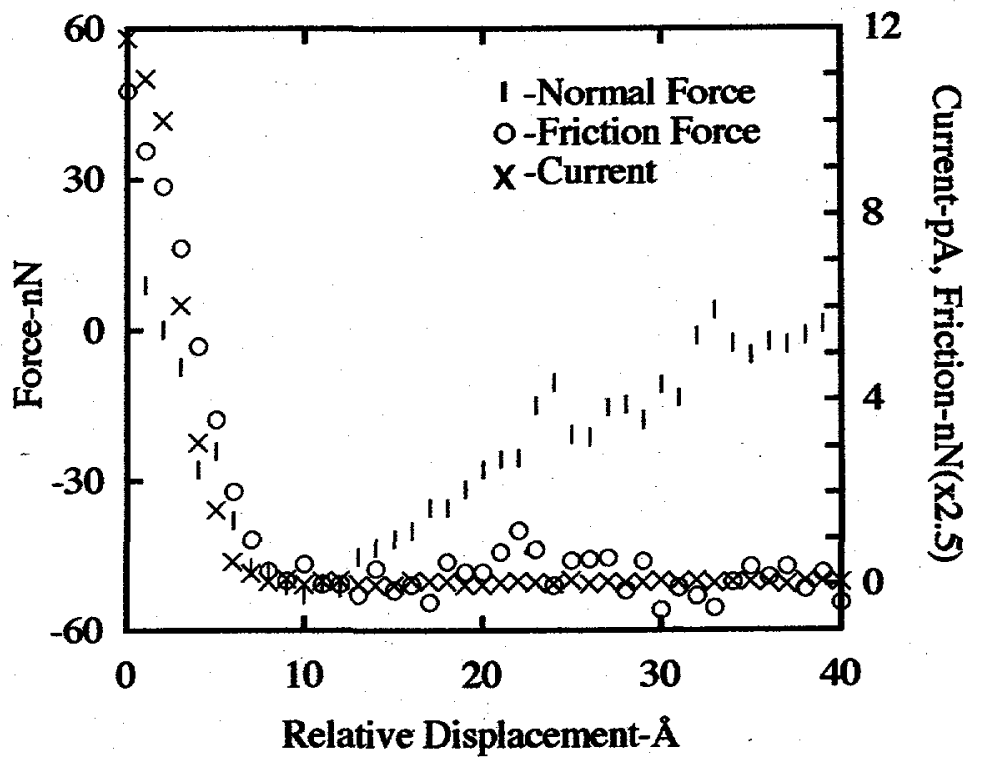

Fig. 3, Son, Kim \& Houston 


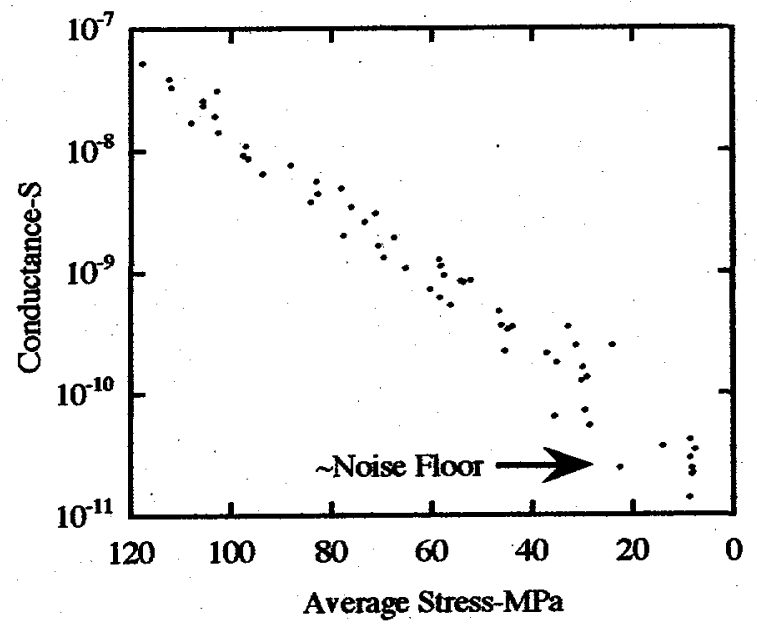

Fig. 4 Son, Kim and Houston 